\title{
The Plant-Stress Metabolites, Hexanoic Aacid and Melatonin, Are Potential "Vaccines" for Plant Health Promotion
}

\author{
Anne J. Anderson ${ }^{1}$ and Young Cheol Kim ${ }^{2 *}$ \\ ${ }^{1}$ Department of Biological Engineering, Utah State University, Logan, UT 84322, USA \\ ${ }^{2}$ Department of Applied Biology, College of Agriculture \& Life Sciences, Chonnam National University, Gwangju \\ 61186, Korea \\ (Received on January 28, 2021; Revised on August 29, 2021; Accepted on August 31, 2021)
}

A plethora of compounds stimulate protective mechanisms in plants against microbial pathogens and abiotic stresses. Some defense activators are synthetic compounds and trigger responses only in certain protective pathways, such as activation of defenses under regulation by the plant regulator, salicylic acid (SA). This review discusses the potential of naturally occurring plant metabolites as primers for defense responses in the plant. The production of the metabolites, hexanoic acid and melatonin, in plants means they are consumed when plants are eaten as foods. Both metabolites prime stronger and more rapid activation of plant defense upon subsequent stress. Because these metabolites trigger protective measures in the plant they can be considered as "vaccines" to promote plant vigor. Hexanoic acid and melatonin instigate systemic changes in plant metabolism associated with both of the major defense pathways, those regulated by SA- and jasmonic acid (JA). These two pathways are well studied because of their induction by different microbial triggers: necrosis-causing microbial pathogens induce the SA pathway whereas colonization by beneficial microbes stimulates the JA pathway. The plant's responses to the two metabolites, however, are not identical with a major dif-

\footnotetext{
*Corresponding author.

Phone) +82-62-530-2071, FAX) +82-62-530-0208

E-mail) yckimyc@jnu.ac.kr

Handling Editor : Eric Johnson

(c) This is an Open Access article distributed under the terms of the Creative Commons Attribution Non-Commercial License (http:// creativecommons.org/licenses/by-nc/4.0) which permits unrestricted noncommercial use, distribution, and reproduction in any medium, provided the original work is properly cited.
}

Articles can be freely viewed online at www.ppjonline.org. ference being a characterized growth response with melatonin but not hexanoic acid. As primers for plant defense, hexanoic acid and melatonin have the potential to be successfully integrated into vaccination-like strategies to protect plants against diseases and abiotic stresses that do not involve man-made chemicals.

Keywords : growth responses, induced systemic tolerance, priming, vaccination

Plants have adapted to survive through extremes of abiotic stresses, even global extinctions, and with competition from many other organisms, some of which injure or cause disease (Cascales-Miñana and Cleal, 2014; McElwain and Punyasena, 2007). Plant health requires sufficient nutrition not only for growth but also for defense against an array of challenges, from insects and nematodes to microbial pathogens as well as a range of abiotic stresses (Agrios, 2005). Consequently, plant defense is multifaceted and includes preformed physical defenses as well as mechanisms that are induced (Agrios, 2005; Osbourn, 1996; Zdor and Anderson, 1992). Methods to induce plant defenses effectively but with limited nontarget effects are desired to maximize yields of quality food products.

Control of human disease sometimes involves vaccination; the Center for Disease Control, USA, defines a vaccine as "a product that stimulates a person's immune system to produce immunity to a specific disease, protecting the person from that disease." Kuć (1982) used the term "immunization" to describe how restricted infection of a plant host with pathogenic fungi, bacteria, or viruses offers systemic protection to plants against subsequent challenges. Their studies illustrate that initial challenge or "immunization" at a sublethal level, followed by a booster 
inoculation, protects cucumber, watermelon, and muskmelon throughout the growing season from a variety of pathogenic challenges (Kuć, 1982). This result is an example of "priming", a term for a plant protective strategy where the priming agent conditions the plant to respond faster and to a greater extent upon subsequent pathogen attack to lessen disease (Conrath et al., 2015). Priming is advantageous in that there is limited energy expenditure for the plant until challenged by a stress (Martinez-Medina et al., 2016). By comparison to the human scenario, the compounds that induce priming in the plant can be regarded as plant "vaccines." Their interaction with the plant boosts immunity in response to subsequent pathogenic stresses. However, plant vaccination with these metabolites also is effective against abiotic stresses of temperature, drought and salinity.

The potential for plant immunization for pathogen control through plant treatments with "vaccines" of diverse structures is already a topic in the literature. For instance, oligochitosans are described as vaccines because of their stimulation of plant immunity (Yin et al., 2010). The paper "Using green vaccination to brighten the agronomic future" focusses on volatiles, chemicals and beneficial microbes as vaccines of promise (Luna, 2016). This theme is updated by Quintana-Rodriguez et al. (2018) in a lively discussion of the role of elicitors, and molecular patterns (pathogenassociated molecular patterns, microbial associated molecular patterns or damage-associated molecular patterns [DAMPs]) as vaccines. Crude plant extracts and volatile organic chemicals also are cited for their roles in defense induction (Quintana-Rodriguez et al., 2018). This activity of plant extracts is interesting because of other early papers where combinations of polysaccharide fungal elicitors with plant wall pectic fractions show synergism in activation of plant defense (e.g., Tepper and Anderson, 1990).

Quintana-Rodriguez et al. (2018) argue that DAMPs that function through a priming mechanism constitute the best type of vaccine because they limit the energy expenditure of the plant until it is challenged by a pathogen. Other recent papers focus on various RNA strategies to immunize the plant against viral diseases (Gago-Zachert et al., 2019; Niehl et al., 2018). With the need to modify agricultural practices to maintain and regenerate soils while providing quality plant-based foods and commodities, it is timely to review the activities of hexanoic acid (HA) and melatonin as examples of plant-based "vaccines" because they prime the plant for immunity. Both of these compounds can be viewed by the public as "natural" or "green" activators. They are present in human food sources and thus are known to be tolerated by human metabolism. Melatonin can easily be purchased by the public over the counter and both products are viable commercial entities so that they are easily available. Their exogenous applications can be regarded as biomimicry allowing the compounds to be already present when the plant is stressed.

The metabolites, HA and melatonin, share the priming effect with several synthetic chemicals. A review by Bektas and Eulgem (2014) focuses on such synthetic primers, including 2,6-dichloroisonicotinic acid (INA). Other compounds, benzo-(1,2,3)-thiadiazole-7-carbothionic acid Smethyl ester (BTH) and $\beta$-aminobutyric acid (BABA), are highlighted in a 2017 review (Mauch-Mani et al., 2017). These compounds prime activation of the plant's defense through the salicylic acid (SA) defense pathway (Conrath, 2009; Conrath et al., 2015).

\section{Hexanoic Acid}

HA, also termed caproic acid (Fig. 1), has a simple structure, being a fatty acid of medium chain length (C6). In plants, HA is synthesized from acetate and is related to the C6-family of green leaf volatiles that are produced upon herbivore damage and physical wounding of leaves (Kroumova et al., 1994). These C6-derivatives also act as primers for plant defenses. Some volatiles of plant and microbial origin also have direct inhibitory effects on pathogen growth (Kong et al., 2020; Kroumova et al., 1994). Thus, the effects of exogenous applications of HA may be boosted by downstream activation of further defense measures native to the plant tissues.

Not only is HA water soluble (about $1 \mathrm{~g} / 100 \mathrm{ml}$ ), allowing mobility in water streams such as in soil pore water, but $\mathrm{HA}$ also is weakly volatile ( 21 pascals at $25^{\circ} \mathrm{C}$ ), facilitating its diffusion through pore spaces in soil and to the atmosphere. Consequently, HA could exert a three-dimensional impact in the environment, independent of water availability to promote defensive measures in adjacent plants.

Volatiles are recognized as important contributors in plant-plant and plant-microbe communication. Although $\mathrm{HA}$ also is produced and metabolized by microbes (Han et al., 2018), the extent to which plant-associated microbes contribute to exogenous HA to influence plant metabolism is not resolved. Exogenous HA, such as through purposeful applications or as part of the volatile cocktail from plant-associated microbes, is thought to enter the plant cell by diffusion with subsequent conversion to its acyl-CoA derivative, and this form could be the metabolite active in triggering plant defense (Llorens et al., 2016). Exogenous applications of ${ }^{13} \mathrm{C}$-labeled HA to citrus roots do not result in detection of the labelled metabolite in the shoot tissues. However, metabolic changes are observed in the shoots; 
treatment with HA alone enhances production of other volatiles, E-2-heptenal and Z-3-hexenol, intermediates of the alpha linoleic pathway. Analysis of ten potential pathways connected with plant defense reveals HA alone activates the linoleic pathway and diterpenoid synthesis (Llorens et al., 2016). However, all ten pathways are activated in HA-treated tissues upon challenge by a pathogen, demonstrating a strong priming effect where defense potential is magnified (Llorens et al., 2016). Although there must be an active signal transduction system accounting for the systemic effects following HA treatments, the mechanism is not yet resolved outside of potential activation by plantproduced volatiles.

\section{Involvement of Both Jasmonic Acid and SA De- fense Pathways in HA-Primed Responses}

HA primes responses that protect plants from viral, bacterial, and fungal diseases (Aranega-Bou et al., 2014), as well as insects (López-Galiano et al., 2017). The defense mechanisms triggered by HA are orchestrated by several plant growth regulators, including those regulated by SA and jasmonic acid (JA). This finding is interesting because these defense pathways can be differentially activated; the SA pathway is initiated by challenge with microbes causing necrosis whereas the JA pathway occurs with coloniza- tion by beneficial microbes (Ton and Mauch-Mani, 2004). For example, levels of HA in root exudates (Kroumova et al., 1994) increase upon root colonization by a biocontrolactive strain of Bacillus cereus (Wang et al., 2019) showing a feed forward effect.

Crosstalk between the JA- and SA-defense pathways exists, and there are situations where these two induced responses are markedly antagonistic (Caarls et al., 2015; Kunkel and Brooks, 2002; Thaler et al., 2002). However, as evinced in the following discussions, the HA-primed responses involve traits characteristic of both the JA- and SA-defense pathways (Scalschi et al., 2013). Additionally, transcriptome analysis illustrates that other plant growth regulators, auxin and ethylene, may be involved in HAprimed responses to subsequent challenge. For example, expression of the gene encoding 1-aminocyclopropane1-carboxylate oxidase, an enzyme required for synthesis of the growth regulator ethylene, is a result of HA priming in tomato upon infection by the necrosis-causing fungal pathogen, Botrytis cinerea (Finiti et al., 2014).

HA treatments trigger a multilayered approach to plant protection, reducing the likelihood of an easy path to pathogen resistance. The broad changes in metabolism (Fig. 1), are an expected consequence of HA-primed defenses being conditioned by several plant growth regulators. Changes in the plant important to defense against microbial pathogens

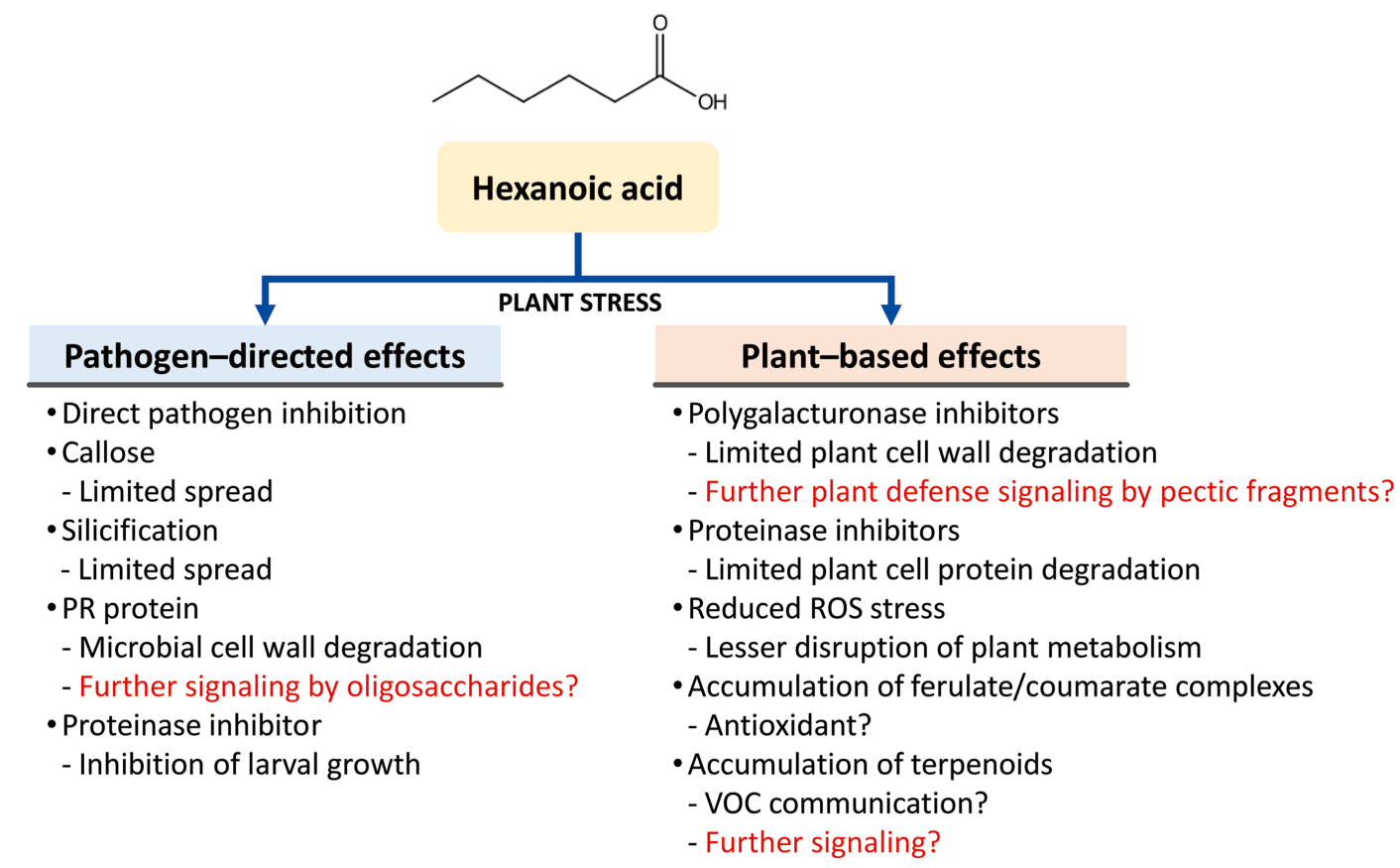

Fig. 1. Outcomes of hexanoic acid priming in plants include responses that directly challenge the pathogen as well as change plant metabolism to boost resistance to pathogens effects. PR, pathogenesis-related; ROS, reactive oxygen species; VOC, volatile organic compounds. 
include the generation of physical blocks and other impediments to pathogen growth, as well as promotion of additional traits that affect plant health (Fig. 1).

HA-primed responses limiting pathogen entry into plant tissues include deposition of callose, a $\beta$-1,3-glucan polymer, at the plant cell wall at the challenge site. The formation of this mechanical barrier is implicated in resistance in several pathosystems: in tomato infected with $B$. cinerea (Finiti et al., 2014); in citrus challenged by the brown spot fungus (Alternaria alternate) (Llorens et al., 2013) or by the bacterium causing citrus canker (Xanthomonas citri) (Llorens et al., 2015). In melon inoculated with melon necrotic spot virus, callose deposition reduces entry of the virus into the phloem, thus lessening viral spread from the initial infection site (Fernandez-Crespo et al., 2017). With other pathogens, callose may limit the spread of damaging enzymes or toxins, to minimize disease symptoms in the plant.

Activation of callose formation is a key part of the protective response against necrotrophs triggered by the synthetic primer, BABA (Ton and Mauch-Mani, 2004). An intimate association between callose formation and another mechanism to boost plant cell wall integrity, silicification, is found in silica-accumulating plants, such as horsetail, and additionally in pathogen-challenged plants (Guerriero et al., 2018). The finding that sites for silica deposition correlate with arabinogalactan-ferulate complexes in the plant cell wall, suggests a connection between silicification and ferulate metabolism (Soukup et al., 2017). This interaction could in part account for the enhanced production of phenolic acids observed in HA-treated melon (FernándezCrespo et al., 2017). Induced silicification also is associated with the action of the synthetic primers, INA and BTH (Kauss et al., 2003). Changes in deposition in the plant cell wall highlights the importance of bioavailable Si in induced disease resistance mechanisms of plants (Epstein, 1999).

Another effect of HA treatment is to reduce the accumulation of reactive oxygen species (ROS) otherwise occurring upon pathogen challenge. Lowering of ROS occurs because HA primes for enhanced activities of peroxidases, and enzymes in the glutathione-ascorbate cycle (Scalschi et al., 2013). Reduced ROS stress is discussed as being a primary effect of beneficial HA treatments (Aranega-Bou et al., 2014). However, the key responses accounting for resistance are likely to be dependent on the pathosystem. For instance, HA-treated citrus shows enhanced production of polygalacturonase inhibitors (Fernandez-Crespo et al., 2017), which should minimize plant cell wall destruction by pectin-degrading enzymes produced by pathogens (da Silva et al., 2002; Isshiki et al., 2001). Simultaneously the inhibitors could promote the formation from the pectins of the oligogalacturonides, which themselves are defense inducers (Ryan and Farmer, 1991). Similarly, HA induction of proteinase inhibitors may be instrumental in controlling pathogens that use proteinases as virulence factors, such as $B$. cinerea (Finiti et al., 2014). The accumulation of proteinase inhibitors additionally would impair growth of larval infestations and thus be very important in control of insect pests (López-Galiano et al., 2017).

Metabolite and transcript profiling of HA-treated citrus challenged with $A$. alternata reveal extensive reprograming of carbon metabolism (Llorens et al., 2016). HA influences primary metabolism by increasing flux through the pentose phosphate pathway which supplies the erythrose4-phosphate used in the shikimic acid pathway for phenolic production. These metabolic changes possibly account for the elevated production of ferulic and coumaric complexes observed in HA-treated melon plants (Fernandez-Crespo et al., 2017) and tobacco cells (Djami-Tchatchou et al., 2017). The changes in levels of phenolics are consistent with up-regulation of genes encoding enzymes involved in aromatic synthesis, such as phenylalanine ammonia lyase (PAL) (Djami-Tchatchou et al., 2017; Llorens et al., 2016). A novel finding is the stimulation by HA of the mevalonic acid pathway, leading to synthesis of a variety of volatile terpenoids. These volatile organic compounds may also play a role in plant defense by regulating another group of defense proteins through the expression of genes encoding pathogenesis-related (PR) proteins as well as PAL (Quintana-Rodriguez et al., 2018; Llorens et al., 2016).

Additionally, HA treatment leads to the accumulation of JA precursors, of which the most prevalent is 12-oxophytodienoic acid (OPDA). OPDA has signaling functions independent of JA, including redox balancing that occurs in part through promotion of cysteine synthesis to boost glutathione production (Park et al., 2013). The initial step in this process is the binding of OPDA to a JA-receptor protein in the chloroplast, cyclophilin 20-3 (CYP20-3), to activate enzymes for cysteine synthesis (Park et al., 2013). As illustrated in Fig. 1, HA primes for multilayered plant defense functions upon pathogen challenge, thus immunizing the plant against varied types of pathogens.

A role for the SA pathway in the responses to HA-vaccination is supported by HA-primed responses in tomato because expression of the SA pathway-marker gene, PRla, is induced when the HA-treated plant is challenged with Botrytis (Finiti et al., 2014). Similarly, HA primes expression of pathogenesis-related proteins from the genes $P R 1$ and $P R 5$ specific to SA regulation upon infection with $P$. syringae. Strong expression of $P R 1$ is found in HA-treated 
cultured tobacco cells in the absence of external stresses (Djami-Tchatchou et al., 2017), although here it is possible that the culturing of the plant cells provides the stress enabling HA to act as a primer. These cells, when treated with $\mathrm{HA}$, also up-regulate a gene encoding the nonexpressor of pathogenesis-related genes 1 (NPR1), the redox-sensitive major regulator of the SA-defense pathway. However, with the tomato- $P$. syringae pathosystem, the genes most strongly primed by HA are those encoding protease inhibitors, responses attributed to activation of the JA-defense pathway. Consistent with the involvement of multiple pathways in HA-related immunization is the finding that the combination of OPDA and SA activation, both induced by HA treatment, correlates with rapid callose deposition (Fernández-Crespo et al., 2017).

\section{Signaling Factors Involved in HA-Primed Re- sponses}

The mechanisms by which HA exposure alters the expression of an array of protective genes remain unclear. Fig. 2 illustrates known and potential molecular signaling events triggered by HA priming, responses that account for the involvement of both SA- and JA- defense pathways. The involvement in HA-priming of the transcription factors, WRKY33 and WRKY53 (Finiti et al., 2014; López-Galiano et al., 2017), supports this view; WRKY53 is a redoxsensitive transcription factor associated with SA-based resistance to $P$. syringae, whereas WRKY33 is linked to the JA defense network (Hu et al., 2012). Generation of novel and small microRNAs occurs in tomato with HA exposure (Lopez-Galiano et al., 2017). Interestingly, some of these microRNAs overlap with responses to drought; thus, these regulatory microRNAs may be associated with the tolerance generated by activation of the JA pathway (Riemann et al., 2015).

Epigenetic changes of acetylation and methylation at specific sites on histones that bind to gene sequences, DNA modification, and chromatin remodeling are also likely to be involved in HA priming (Ding and Wang, 2015; Ramirez-Prado et al., 2018). In Arabidopsis, systemic acquired resistance triggered by pathogenic $P$. syringae is passed to new plant generations (Luna et al., 2012), with the offspring showing greater resilience to this pathogen. Expression of SA-pathway defense genes is associated with acetylation of histone $\mathrm{H} 3$, specifically at lysine 9, affecting binding to the genes' promoters, whereas repression of JA pathway marker genes is associated with methylation of histone H3. DNA methylation also is required for transgenerational resistance. For rice, priming with methyl jasmonate (MeJA) changes DNA methylation and modifies the histones associated with the promoter of a defense gene (Laura et al., 2018). In tomato challenged by B. cinerea, a methylated histone, $\mathrm{H} 3 \mathrm{~K} 4 \mathrm{me} 3$, associates with an exon in the gene encoding the defense pathway transcription factor WRKY53 (Crespo-Salvador et al., 2018). This methylated histone also is associated with binding at the promoter of WRKY53 in the Arabidopsis- $P$. syringae pathosystem (Jaskiewicz et al., 2011). The relevance of epigenetic changes in HA priming awaits further investigation, as does the finding of whether protective effects of HA exposure are continued to the next plant generation.

\section{Melatonin}

Melatonin has multiple beneficial effects in plants (Fig. 3 ), observed in response to foliar and root applications, as well as in response to changes in naturally occurring levels. Indeed, Arnao and Hernández-Ruiz (2019a) coin the term "master regulator" for this metabolite and raise the question of its role as a plant hormone (Arnao and Hernández -Ruiz, 2020a). This concept of melatonin as a master regulator is strengthened by discussion in Arnao and Hernández-Ruiz (2020b) of melatonin effects on plant hormones from their

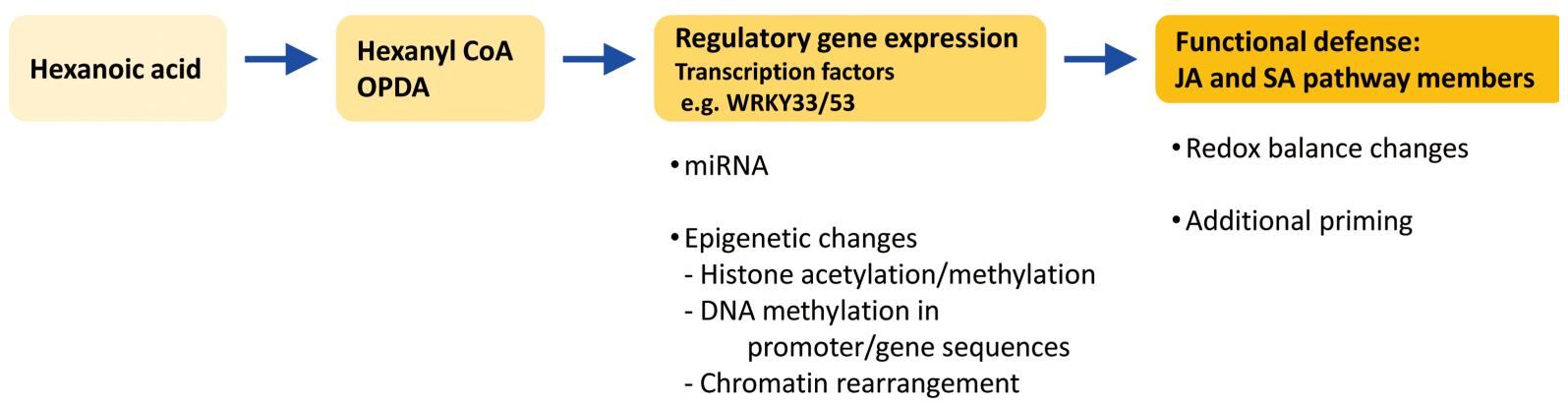

Fig. 2. Signaling events in plants in response to hexanoic acid priming. Hexanyl CoA may be the active molecule derived from hexanoic acid. 12-oxo-phytodienoic acid (OPDA) is the precursor of jasmonic acid (JA) and metabolic changes in the plant cells characteristic of the JA-defense pathway are associated with the hexanoic acid-primed plant responses. SA, salicylic acid. 
synthesis/degradation to their signaling activity. These hormones include the growth regulators, indole acetic acid, gibberellic acid and cytokinins, as well as the stress regulators, jasmonate, brassinosteroids, polyamines, abscisic acid and SA.

Similar to HA, melatonin alleviates stress caused by ROS and reactive nitrogen species (RNS) and through activating the expression of genes encoding defensive proteins provide protection against microbial diseases (Arnao and Hernández-Ruiz, 2019b). Plants subjected to both stress and melatonin treatment show synergetic changes in gene expression compared with plants subjected to stress or melatonin treatment alone; these responses are characteristic of primer activity from melatonin applications.

Furthermore, melatonin helps regulate plant growth and development (Arnao and Hernandez-Ruiz, 2019a). These effects may relate in part from improved nutrition such as balancing balancing Fe levels, as observed in cucumber (Ahammed et al., 2020). Consequently, melatonin is often termed a plant "biostimulant." In this paper, the priming effect of melatonin on plant immunity is highlighted among its varied beneficial effects.

\section{Melatonin Biosynthesis}

Melatonin, $N$-acetyl-5-methoxytryptamine (Fig. 3), is found in plants even in the absence of apparent environmental stress. Levels vary with the plant tissue (e.g., seeds and fruits vs. shoots) at concentrations up to $50 \mu \mathrm{g} / \mathrm{g}$ although most concentrations are of the order of ng/g (Arnao, 2014; Debnath et al., 2019; Sharif et al., 2018). Melatonin is produced from the amino acid, tryptophan, through a multistep - process, involving tryptamine and serotonin as intermediates (Arnao and Hernández-Ruiz, 2020b; Back et al., 2016; Hardeland, 2016). Thus, melatonin synthesis, unlike that of HA which is acetate/lipid based, requires integration with $\mathrm{N}$ metabolism in the plant.

Increased biosynthesis of melatonin is observed in plants in response to stress including challenge by avirulent bacteria and pathogens (Lee and Back, 2017). Changes in redox cell signaling involving ROS and nitric oxide, produced by the stress, triggers melatonin production (Li et al., 2019). Biosynthesis from tryptophan requires increases in carbon flux through the pentose phosphate pathway to the shikimic acid pathway-metabolite, chorismate, from which the

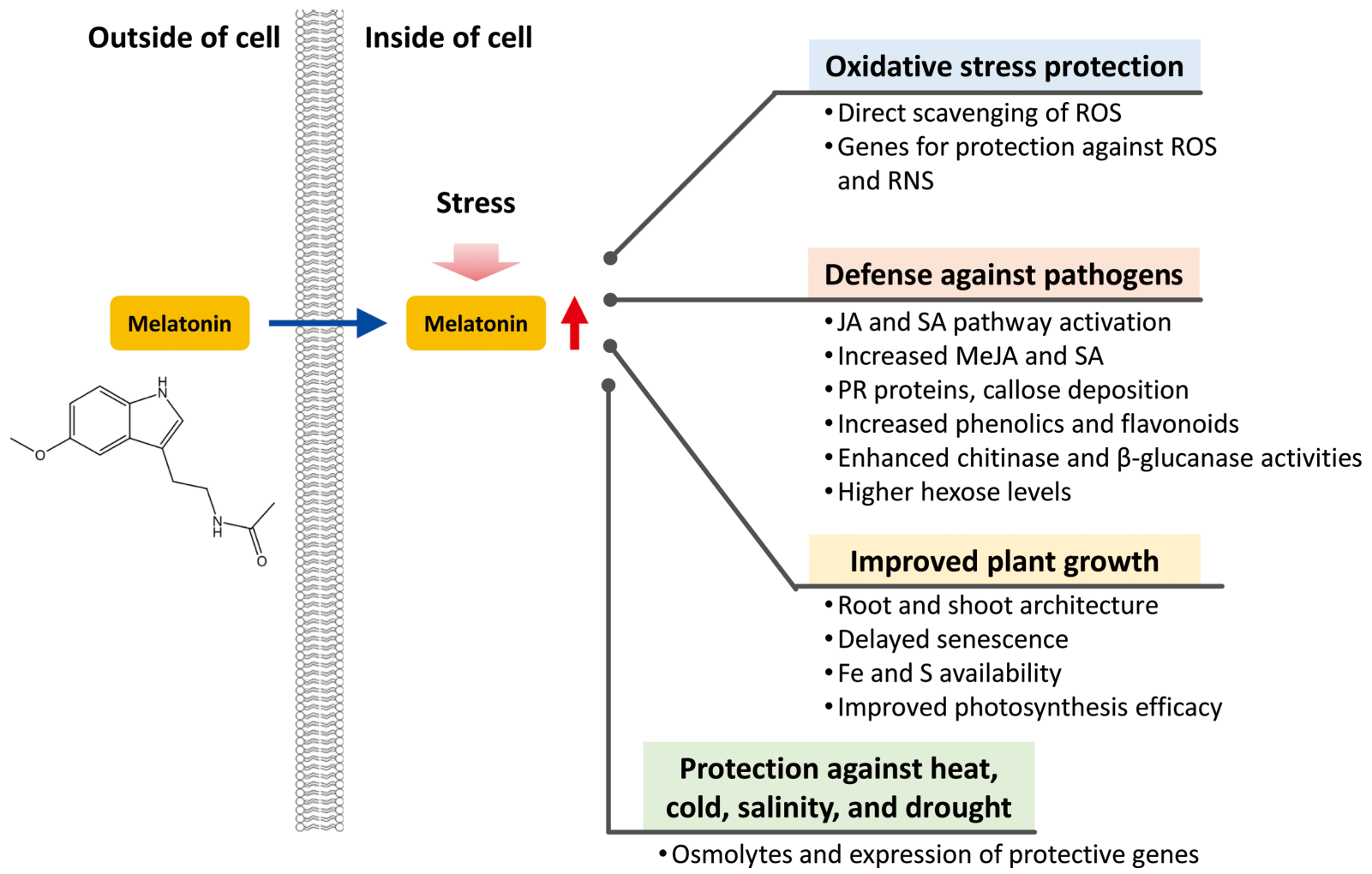

Fig. 3. Beneficial outcomes from melatonin treatment. Exogenous application of melatonin induces physiological changes in plant cells that provide protection against reactive oxygen and nitrogen species (ROS and RNS). Plant level responses result in protection from abiotic stress and pathogen challenge, as well as improved plant growth. JA, jasmonic acid; SA, salicylic acid; MeJA, methyl jasmonate; PR, pathogenesis-related. 
amino acid precursor for melatonin biosynthesis is derived (Hernández-Ruiz and Arnao, 2018).

Both chloroplasts and mitochondria serve as sites for melatonin biosynthesis (Tan et al., 2013), although biosynthesis may also occur in the cytoplasm (Back et al., 2016). Different locations of melatonin accumulation within the plant may depend on which of its many functions are significant for that tissue. Melatonin biosynthesis within the chloroplast and mitochondria may be a legacy of the evolution of these structures from bacterial endosymbionts that had already evolved pathways to produce melatonin because of its antioxidant activity (Tan and Reiter, 2020; Zhao et al., 2019). Subsequently, over the course of evolutionary time, the roles played by melatonin in plants likely have diversified.

\section{Beneficial Effects of Melatonin}

The main functions of melatonin in plants, as depicted in Fig. 3, are in providing protection against oxidative stress, boosting protection against biotic and abiotic challenges, and regulating growth (Arnao and Hernández-Ruiz, 2019a, 2019b; Debnath et al., 2019; Hardeland, 2016; Lee et al., 2014; Moustafa-Farag et al., 2019, 2020; Shi et al., 2016; Tan et al., 2015). Melatonin combats oxidative stress from ROS and RNS through different strategies. One mechanism involves direct structural modification of the melatonin molecule by hydroxyl radicals, superoxide anions and singlet oxygen; this process removes these harmful reactive species (Hardeland, 2016). The oxidative modifications to melatonin occur largely through free radical cascade reactions so that the changes are not enzymatically catalyzed (Hardeland, 2016; Tan et al., 2015). Some of the melatonin derivatives produced in the free radical cascade also have protective properties against oxidative stress (Tan et al., 2015). Stress induction of melatonin biosynthesis coupled with melatonin increasing the expression of genes encoding protective proteins (Tan et al., 2015) very effectively scavenges reactive oxygen and nitrogen species to lessen their damaging effects (Arnao and Hernández-Ruiz, 2019b). It is this innate activity of the molecule that probably accounts for melatonin's presence in seeds, where protection against oxidative damage is needed at low water potential to support enzymatic activity. Similarly, melatonin is protective in the chloroplasts and mitochondria, alleviating the effects of aberrant electron flow in electron transfer chains (Martinez et al., 2018; Tan et al., 2013). Indeed, the plant protection conferred by melatonin against heat stress is associated with changes in responses to oxidative stress (Buttar et al., 2020). Thus, melatonin's ability to control oxidative stress has widespread beneficial consequences (Fig. 3).

\section{Protection against Abiotic Stresses}

Under abiotic stress, melatonin may play a major role in protecting photosynthetic capacity through the reduction of ROS damage (Sharma and Zheng, 2019). Melatonin application improves growth of tomato subjected to extremes of salinity and temperature (Martinez et al., 2018). Measurements of $\mathrm{CO}_{2}$ assimilation, transpiration rate, stomatal conductance and photosystem II efficacy show maintenance of photosynthesis. This protection correlates with less accumulation of hydrogen peroxide and oxidized lipids with melatonin treatment of plants stressed by heat and salinity (Martinez et al., 2018). Examination of gene transcription and enzyme activities finds little effect of melatonin application alone but activation of protective responses upon abiotic stress. Similar observations are reported for tomato under salinity stress when treated with melatonin: ROS accumulation is decreased and enzymes for ROS protection modified (Siddiqui et al., 2019). They also find the osmolyte, proline, to be highest in saline-stressed plants when treated with melatonin; this and other osmolytes, such as sugars, would enhance plant cell integrity and enzymatic function under stress. Additionally, maintenance of the photosynthetic ability is promoted by the higher levels of the primary $\mathrm{CO}_{2}$-fixing enzyme, ribulose 1,5-bisphosphate carboxylase-oxygenase, resulting in continued accumulation of carbohydrates in stressed, melatonin-treated plants.

Maintenance of chloroplast photosynthetic function and structural integrity is proposed to be a major factor in the melatonin-induced resilience of tomato to cold stress (Yang et al., 2018). For watermelon, transcript profiling additionally reveals increased expression of novel protective genes specific for cold stress tolerance. Applications of melatonin to roots provides protection in shoots and vice versa suggesting that systemic transport can occur (Li et al., 2017). The observation that melatonin increases in the xylem when plants with melatonin-treated roots are cold shocked suggests that the metabolite itself is mobile in the plant (Li et al., 2017). With exogenous applications, uptake and subsequent vascular transport could explain the observed systemic protective effects (Li et al., 2017). Melatonin when applied to roots alone induces very little change in expression of cold-stress defense genes in leaves until the plant is cold shocked (Li et al., 2017). These observations are consistent with a priming action of melatonin to alleviate temperature stress problems in plants.

Melatonin treatment also protects against drought. The binding of melatonin to a specific receptor initiates sto- 
matal closure (Wei et al., 2018); currently this is the only melatonin receptor identified in plant cells. In Arabidopsis thaliana, melatonin is bound to the receptor CAND2 protein in the receptor complex, CAND2/PMTR1, located in the plasma membrane. Hydrogen peroxide production by a NADPH-oxidase is triggered through $G$ protein interactions with the loaded melatonin-receptor complex. The hydrogen peroxide burst signals altered flux of $\mathrm{Ca}$ and $\mathrm{K}$ ions, promoting stomatal closure (Wei et al., 2018). A second but undefined mechanism for drought protection is indicated from studies in Brassica where the maintenance of root growth during drought by melatonin might allow continued water uptake (Dai et al., 2020). The promotion of lateral root formation by melatonin would increase the potential for the root to access soil water and contribute to drought tolerance in melatonin-treated plants (Zhang et al., 2014).

As anticipated, given the ability of melatonin to ameliorate ROS stress, melatonin treatments protect plant cells against microbial pathogens. Fig. 3 summarizes the major research-supported mechanisms by which melatonin confers resistance to pathogenic bacteria, fungi, and viruses; the range of pathosystems for which melatonin offers protection is extensive (Moustafa-Farag et al., 2020). Although little is known in depth of the mechanisms underlying protection against viruses, the combination of JA-, SA-, and ethylene-regulated defense pathways is cited as being responsible for melatonin control of bacterial and fungal pathogens (Moustafa-Farag et al., 2020). These same possibilities also apply to plant protection afforded by HA treatments (Fig. 4).

Molecular evidence from transcription studies in Arabidopsis suggests that melatonin alone enhances expression of defense genes associated with abscisic acid, ethylene, SA, and JA regulation (Weeda et al., 2014). A 100 pM dose down-regulates over 51 genes and upregulates 30 genes; a higher dose, $1 \mathrm{mM}$, results in greater changes with expression from 1,308 genes being affected. These findings illustrate that gene expression in plants responds to melatonin alone, although not to an overwhelming degree as is characteristic of the plant's detection of a priming agent without a stress.

Biosynthesis of melatonin in Arabidosis is stimulated in the incompatible interaction with an avirulent $P$. syringae isolate (Lee and Back, 2017) through a process requiring both hydrogen peroxide and nitric oxide signaling that likely results from the pathogen attack. This plant-microbe interaction activates the SA defense pathway with anticipated participation of the mitogen-activated protein kinase cascade, feeding into MAPK3 and MAPK6 activation for regulating defense gene expression (Lee and Back, 2017). Exogenous applications of melatonin also initiate a burst of superoxide anions followed by peaks of hydrogen peroxide and nitric oxide leading to increased plant biosynthesis of melatonin and triggering of the SA-defense pathway (Li et al., 2019). These changes are proposed to be instrumental in control by melatonin treatments of postharvest lesions in tomato fruits caused by the necrotic fungus, $B$. cinerea $(\mathrm{Li}$ et al., 2019). The findings of a cascade of events leading to SA-regulated defense as a mechanism for change in the transcriptome is observed in $A$. thaliana following melatonin treatment (Weeda et al., 2014).

Although a role for the SA pathway in defending against B. cinerea in tomato fruits is supported (Li et al., 2017), other research (Liu et al., 2019) supports that the JA pathway is activated simultaneously. Here the plant responses to melatonin and HA are different because melatonin treatment increases MeJA levels, whereas HA treatment tends to raises concentrations of the JA-leucine complex (Fernández-Crespo et al., 2017). Other melatonin-induced changes in gene expression support the activation of the JA pathway, such as enhanced expression of enzymes involved in JA-synthesis, LoxD and protease inhibitor II (Weeda et al., 2014). Melatonin increases the transcription of genes encoding the PR proteins, chitinases and $\beta$-glucanases, changes that foretell of possible degradation of chitin and glucans in the cell walls of fungal pathogens. Such fungal wall degradation would release short oligosaccharides (chitosan and oligoglucans) which act as elicitors of defense to diversify and intensify resistance strategies.

Melatonin treatment, like HA (Fig. 4) results in deposition of the protective cell wall polymer callose in Arabidopsis upon challenge with a virulent isolate of $P$. syringae (Zhao et al., 2015). Additionally, the levels of phenolics and flavonoids increase in response to melatonin (Simlat et al., 2018), although there are no reports for enhanced lignification, so that the roles of these compounds remain unresolved. Currently, there are no published works relating melatonin to plant cell wall silicification, which, as discussed under HA-induced responses, is linked with ferulate complexes in the plant cell wall (Figs. 1 and 4).

The boost in photosynthetic efficiency in Arabidopsis upon melatonin treatment causes increased sucrose flux and hexose accumulation (Sharma and Zheng, 2019). Sugars play roles in cell signaling, operating through three different mechanisms to regulate the expression of genes involved in plant development and defense (Tauzin and Giardina, 2014; Wingler, 2018). For instance, in rice, expression of several PR genes is controlled by sucrose (Gómez-Ariza et al., 2007). However, the extent to which 


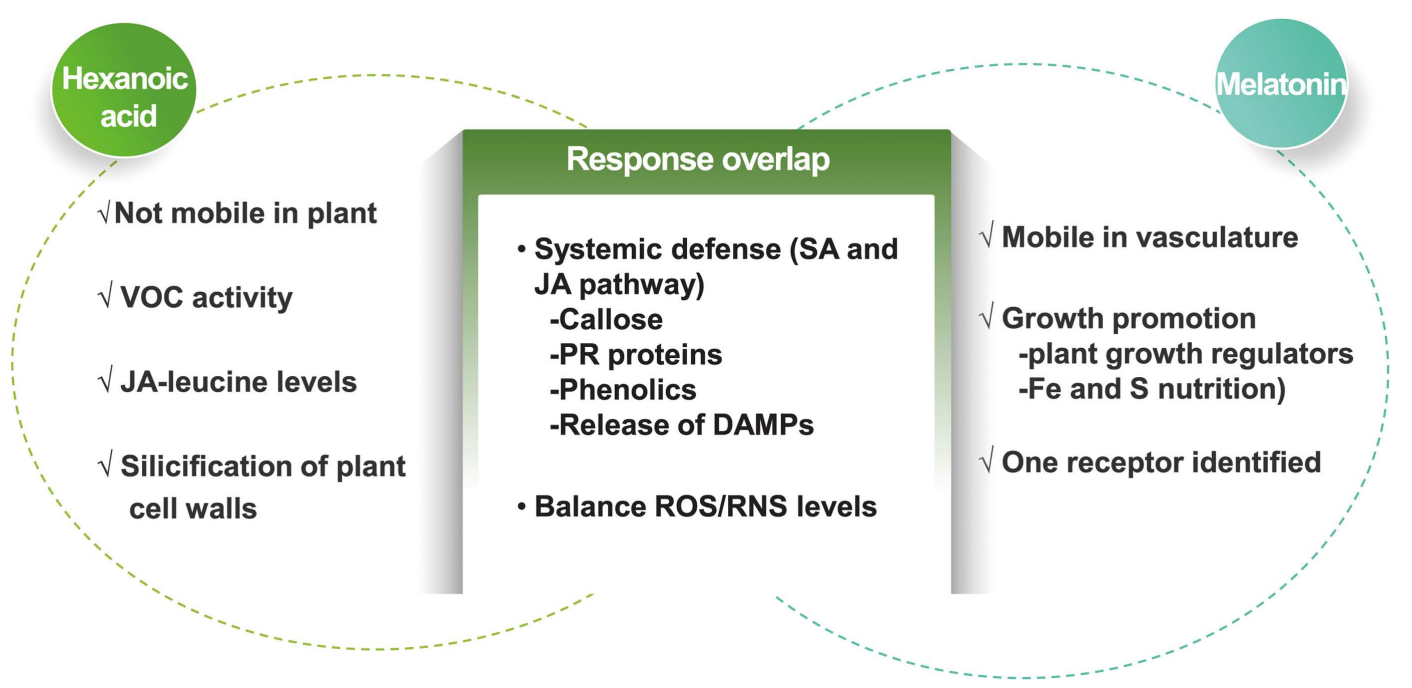

Fig. 4. Comparisons of plant responses to hexanoic acid and melatonin illustrating major similarities and differences in effects. VOC, volatile organic compounds; SA, salicylic acid; JA, jasmonic acid; PR, pathogenesis-related; DAMP, damage-associated molecular pattern; ROS, reactive oxygen species; RNS, reactive nitrogen species.

sugar signaling plays a role in plants' responses to melatonin awaits clarification. The sugars themselves have protective effects as osmolytes and scavengers for ROS.

These findings establish that melatonin regulates the JA and SA defense pathways to enhance plant immunity against microbial diseases. Melatonin acts like a vaccine in priming the plant tissues to reserve the use of these energyexpensive defense pathways in a balanced and constructive manner after the plant is microbially challenged. Priming saves energy, as well as the $\mathrm{C}$ and $\mathrm{N}$ supplies which then would be available for other plant functions, such as growth and development.

\section{Improvement of Plant Growth}

Melatonin induces increased growth of plants at the wholeplant level, with effects on germination, altered root morphology, and delayed senescence (Arnao and HernándezRuiz, 2014) (Fig. 3). Unlike melatonin, HA has not been reported to have any effects on growth and this trait is a major documented difference in the response to the application of these metabolites (Fig. 4). In Stevia, a plant in which germination is difficult to induce, melatonin treatment increases germination rate and seedling mass in a dose-dependent manner (Simlat et al., 2018). The melatonin-treated seedlings have higher levels of phenolics and sugars than the controls lacking treatment. Melatonin may promote primary root growth and formation of lateral roots, depending on dose and plant type (Arnao and HernandezRuiz, 2014); Sharma and Zheng, 2019). Although auxin and melatonin have similarity in their structure, melatonin affects root architecture by mechanisms that are independent of auxin (Wan et al., 2018).

Enhanced growth may be related to improved nutrition in melatonin-treated plants. A study in tomato finds that melatonin enhances sulfur uptake and metabolism resulting in improved resilience to oxidative stress (Hasan et al., 2018). Melatonin also may boost Fe nutrition in Arabidopsis (Wan et al., 2018). Lateral root formation is stimulated by melatonin, and expression of genes that regulate and encode the proteins involved in Fe uptake into the root is increased. Altered Fe transport into roots with melatonin application occurs likewise in cucumber (Ahammed et al., 2020). This process is sensitive to the Fe level because melatonin restricts $\mathrm{Fe}$ transport at high $\mathrm{Fe}$, but enhances root uptake at low Fe bioavailability. The same study found that Fe increases PAL activity, possibly providing the link to the higher levels of phenolics produced in cucumber by melatonin treatments (Ahammed et al., 2020).

Melatonin, and its precursor serotonin, enhance expression of two genes encoding proteins that function in plant cell wall remodeling (Wan et al., 2018). Both melatonin and serotonin accelerate catabolism of the basic amino acids and alanine, while promoting the formation of asparagine, an amino acid that distributes $\mathrm{N}$ throughout the plant. Interestingly, serotonin, but not melatonin, has the potential to improve photosynthetic efficiency by increasing expression of genes with functions in both photosystems (Wan et al., 2018). Clearly, there is a need to assess the potency of serotonin versus melatonin as a biostimulant and a poten- 
tial primer for protective genes. It would also be interesting to examine the roles of sugar sensing and priming in melatonin-induced growth responses. Sugar sensing events are primary regulators of plant growth as well as being involved in expression of defense genes (Wingler, 2018).

Plant productivity may additionally be promoted by melatonin because in addition to promoting growth, melatonin delays senescence, in part because photosynthesis is protected. Findings in kiwifruit leaves (Liang et al., 2018) support that melatonin increases biosynthesis of the chlorophyll $\mathrm{a} / \mathrm{b}$ binding proteins, contributing to prolonged effective photosynthesis. Simultaneously, melatonin improves the anti-antioxidant potential, through the additive effects of increased enzyme activities for ROS destruction (peroxidase and superoxide dismutase) and enhanced activity of the glutathione-ascorbate cycle, and heightened levels of antioxidant flavonoids. Each of these methods for ROSscavenging delay the onset of scenescence.

\section{Summary}

This paper highlights two metabolites, HA and melatonin, produced by plants and microbes, which act as primers for defense pathway activation in plants. Both of these materials are already part of the human diet, and thus may have a public perception as "green" chemicals versus the xenobiotic primers. These metabolites of different molecular structure are both plant stress metabolites, indicating that these are part of a" feedforward" protection process whereby their production enhances resistance in the plant upon subsequent stress, i.e., they act as promoters for plant health. Although their chemical structures are different, plant responses to both metabolites overlap in that they induce layered plant defenses through balanced activation of the SA- and JA-defense pathways (Fig. 4). However, other responses currently are only represented in the literature to be consequences of the exposure of either HA or melatonin (Fig. 4). Direct studies of comparisons of plant responses to HA and melatonin, as well as combination of these priming metabolites, would be very valuable.

The finding that exogenous applications of HA and melatonin stimulate plant immunity to microbial pathogens suggests both metabolites may be regarded as a type of vaccine for plants. They have the potential to protect against a wide variety of plant pathogens and insect pests but also promote tolerance to abiotic stresses. Melatonin treatment differs from HA in that there is evidence of an added advantage of improving plant growth and nutrition (Fig. 3 and 4). The beneficial effects of HA may be integrated with improved plant defense associated with certain probiotic microbes as they colonize plant tissues. The potential of HA and melatonin as vaccines to stimulate plant immunity is one that can be integrated into green solutions to maintain the supply for plant-based foods and products while maintaining healthy soils.

\section{Conflicts of Interest}

No potential conflict of interest relevant to this article was reported.

\section{Acknowledgments}

This work was supported by Korea Institute of Planning and Evaluation for Technology in Food, Agriculture and Forestry (IPET) through Agricultural Machinery/Equipment Localization Technology Development Program, funded by Ministry of Agriculture, Food and Rural Affairs (MAFRA) (321055-05) and previous support from the USDA and NSF from grants to AJA.

\section{References}

Agrios, G. 2005. Plant pathology. 5th ed. Academic Press, Amsterdam, The Netherlands. 953 pp.

Ahammed, G. J., Wu, M., Wang, Y., Yan, Y., Mao, Q., Ren, J., Ma, R., Liu, A. and Chen, S. 2020. Melatonin alleviates iron stress by improving iron homeostasis, antioxidant defense and secondary metabolism in cucumber. Sci. Hortic. 265:109205.

Aranega-Bou, P., de la O. Leyva, M., Finiti, I., García-Agustín, P. and González-Bosch, C. 2014. Priming of plant resistance by natural compounds: hexanoic acid as a model. Front. Plant Sci. 5:488.

Arnao, M. B. 2014. Phytomelatonin: discovery, content, and role in plants. Adv. Bot. 2014:815769.

Arnao, M. B. and Hernández-Ruiz, J. 2014. Melatonin: plant growth regulator and/or biostimulator during stress? Trends Plant Sci. 19:789-797.

Arnao, M. B. and Hernández-Ruiz, J. 2018. Melatonin in its relationship to plant hormones. Ann. Bot. 121:195-207.

Arnao, M. B. and Hernández-Ruiz, J. 2019a. Melatonin: a new plant hormone and/or a plant master regulator? Trends Plant Sci. 24:38-48.

Arnao, M. B. and Hernández-Ruiz, J. 2019b. Melatonin and reactive oxygen and nitrogen species: a model for the plant redox network. Melatonin Res. 2:152-168.

Arnao, M. B. and Hernández-Ruiz, J. 2020a. Is phytomelatonin a new plant hormone? Agronomy 10:95.

Arnao, M. B. and Hernández-Ruiz, J. 2020b. Melatonin as a regulatory hub of plant hormone levels and action in stress situations. Plant Biol. 23:7-19.

Back, K., Tan, D.-X. and Reiter, R. J. 2016. Melatonin biosynthesis in plants: multiple pathways catalyze tryptophan to mela- 
tonin in the cytoplasm or chloroplasts. J. Pineal Res. 61:426437.

Bektas, Y. and Eulgem, T. 2014. Synthetic plant defense elicitors. Front. Plant Sci. 5:804.

Buttar, Z. A., Wu, S. N., Arnao, M. B., Wang, C., Ullah, I. and Wang, C. 2020. Melatonin suppressed the heat stress-induced damage in wheat seedlings by modulating the antioxidant machinery. Plants 9:809.

Caarls, L., Pieterse, C. M. and Van Wees, S. C. 2015. How salicylic acid takes transcriptional control over jasmonic acid signaling. Front. Plant Sci. 6:170.

Cascales-Miñana, B. and Cleal, C. J. 2014. The plant fossil record reflects just two great extinction events. Terra Nova 26:195200.

Conrath, U. 2009. Chapter 9. Priming of induced plant defense responses. In: Advances in botanical research, ed. by L. C. V. Loon, pp. 361-395. Academic Press, Burlington, MA, USA.

Conrath, U., Beckers, G. J. M., Langenbach, C. J. G. and Jaskiewicz, M. R. 2015. Priming for enhanced defense. Annu. Rev. Phytopathol. 53:97-119.

Crespo-Salvador, Ó., Escamilla-Aguilar, M., López-Cruz, J., López-Rodas, G. and González-Bosch, C. 2018. Determination of histone epigenetic marks in Arabidopsis and tomato genes in the early response to Botrytis cinerea. Plant Cell Rep. 37:153-166.

da Silva, A. C. R., Ferro, J. A., Reinach, F. C., Farah, C. S., Furlan, L. R., Quaggio, R. B., Monteiro-Vitorello, C. B., Van Sluys, M. A., Almeida, N. F., Alves, L. M. C., do Amaral, A. M., Bertolini, M. C., Camargo, L. E. A., Camarotte, G., Cannavan, F., Cardozo, J., Chambergo, F., Ciapina, L. P., Cicarelli, R. M. B., Coutinho, L. L., Cursino-Santos, J. R., El-Dorry, H., Faria, J. B., Ferreira, A. J. S., Ferreira, R. C. C., Ferro, M. I. T., Formighieri, E. F., Franco, M. C., Greggio, C. C., Gruber, A., Katsuyama, A. M., Kishi, L. T., Leite, R. P., Lemos, E. G. M., Lemos, M. V. F., Locali, E. C., Machado, M. A., Madeira, A. M. B. N., Martinez-Rossi, N. M., Martins, E. C., Meidanis, J., Menck, C. F. M., Miyaki, C. Y., Moon, D. H., Moreira, L. M., Novo, M. T. M., Okura, V. K., Oliveira, M. C., Oliveira, V. R., Pereira, H. A., Rossi, A., Sena, J. A. D., C. Silva, S., de Souza, R. F., Spinola, L. A. F., Takita, M. A., Tamura, R. E., Teixeira, E. C., Tezza, R. I. D., Trindade dos Santos, M., Truffi, D., Tsai, S. M., White, F. F., Setubal, J. C. and Kitajima, J. P. 2002. Comparison of the genomes of two Xanthomonas pathogens with differing host specificities. Nature 417:459463.

Dai, L., Li, J., Harmens, H., Zheng, X. and Zhang, C. 2020. Melatonin enhances drought resistance by regulating leaf stomatal behaviour, root growth and catalase activity in two contrasting rapeseed (Brassica napus L.) genotypes. Plant Physiol. Biochem. 149:86-95.

Debnath, B., Islam, W., Li, M., Sun, Y., Lu, X., Mitra, S., Hussain, M., Liu, S. and Qiu, D. 2019. Melatonin mediates enhancement of stress tolerance in plants. Int. J. Mol. Sci. 20:1040.

Ding, B. and Wang, G.-L. 2015. Chromatin versus pathogens: the function of epigenetics in plant immunity. Front. Plant Sci.
$6: 675$.

Djami-Tchatchou, A. T., Ncube, E. N., Steenkamp, P. A. and Dubery, I. A. 2017. Similar, but different: structurally related azelaic acid and hexanoic acid trigger differential metabolomic and transcriptomic responses in tobacco cells. BMC Plant Biol. 17:227.

Epstein, E. 1999. Silicon. Annu. Rev. Plant Physiol. Plant Mol. Biol. 50:641-664.

Fernández-Crespo, E., Navarro, J. A., Serra-Soriano, M., Finiti, I., García-Agustín, P., Pallás, V. and González-Bosch, C. 2017. Hexanoic acid treatment prevents systemic MNSV movement in Cucumis melo plants by priming callose deposition correlating SA and OPDA accumulation. Front. Plant Sci. $8: 1793$.

Finiti, I., de la O. Leyva, M., Vicedo, B., Gómez-Pastor, R., LópezCruz, J., García-Agustín, P., Real, M. D. and GonzálezBosch, C. 2014. Hexanoic acid protects tomato plants against Botrytis cinerea by priming defence responses and reducing oxidative stress. Mol. Plant Pathol. 15:550-562.

Gago-Zachert, S., Schuck, J., Weinholdt, C., Knoblich, M., Pantaleo, V., Grosse, I., Gursinsky, T. and Behrens, S.-E. 2019. Highly efficacious antiviral protection of plants by small interfering RNAs identified in vitro. Nucleic Acids Res. 47:9343-9357.

Gómez-Ariza, J., Campo, S., Rufat, M., Estopà, M., Messeguer, J., San Segundo, B. and Coca, M. 2007. Sucrose-mediated priming of plant defense responses and broad-spectrum disease resistance by overexpression of the maize pathogenesis-related PRms protein in rice plants. Mol. Plant-Microbe Interact. 20:832-842.

Guerriero, G., Stokes, I. and Exley, C. 2018. Is callose required for silicification in plants? Biol. Lett. 14:20180338.

Han, W., He, P., Shao, L. and Lü, F. 2018. Metabolic interactions of a chain elongation microbiome. Appl. Environ. Microbiol. 84:e01614-18.

Hardeland, R. 2016. Melatonin in plants: diversity of levels and multiplicity of functions. Front. Plant Sci. 7:198.

Hasan, M. K., Liu, C.-X., Pan, Y.-T., Ahammed, G. J., Qi, Z.Y. and Zhou, J. 2018. Melatonin alleviates low-sulfur stress by promoting sulfur homeostasis in tomato plants. Sci. Rep. 8:10182.

Hernández-Ruiz, J. and Arnao, M. B. 2018. Relationship of melatonin and salicylic acid in biotic/abiotic plant stress responses. Agronomy 8:33.

Hu, Y., Dong, Q. and Yu, D. 2012. Arabidopsis WRKY46 coordinates with WRKY70 and WRKY53 in basal resistance against pathogen Pseudomonas syringae. Plant Sci. 185186:288-297.

Isshiki, A., Akimitsu, K., Yamamoto, M. and Yamamoto, H. 2001. Endopolygalacturonase is essential for citrus black rot caused by Alternaria citri but not brown spot caused by Alternaria alternata. Mol. Plant-Microbe Interact. 14:749-757.

Jaskiewicz, M., Conrath, U. and Peterhänsel, C. 2011. Chromatin modification acts as a memory for systemic acquired resistance in the plant stress response. EMBO Rep. 12:50-55. 
Kauss, H., Seehaus, K., Franke, R., Gilbert, S., Dietrich, R. A and Kröger, N. 2003. Silica deposition by a strongly cationic proline-rich protein from systemically resistant cucumber plants. Plant J. 33:87-95.

Kong, H. G., Song, G. C., Sim, H.-J. and Ryu, C.-M. 2020. Achieving similar root microbiota composition in neighbouring plants through airborne signalling. ISME J. 15:397-408.

Kroumova, A. B., Xie, Z. and Wagner, G. J. 1994. A pathway for the biosynthesis of straight and branched, odd- and evenlength, medium-chain fatty acids in plants. Proc. Natl. Acad. Sci. U. S. A. 91:11437-11441.

Kuć, J. 1982. Induced immunity to plant disease. BioScience 32:854-860.

Kunkel, B. N. and Brooks, D. M. 2002. Cross talk between signaling pathways in pathogen defense. Curr. Opin. Plant Biol. 5:325-331.

Laura, B., Silvia, P., Francesca, F., Benedetta, S. and Carla, C. 2018. Epigenetic control of defense genes following MeJAinduced priming in rice (O. sativa). J. Plant Physiol. 228:166177.

Lee, H. Y. and Back, K. 2017. Melatonin is required for $\mathrm{H}_{2} \mathrm{O}_{2-}$ and NO-mediated defense signaling through MAPKKK3 and OXI1 in Arabidopsis thaliana. J. Pineal Res. 62:e12379.

Lee, H. Y., Byeon, Y. and Back, K. 2014. Melatonin as a signal molecule triggering defense responses against pathogen attack in Arabidopsis and tobacco. J. Pineal Res. 57:262-268.

Li, H., Chang, J., Zheng, J., Dong, Y., Liu, Q., Yang, X., Wei, C., Zhang, Y., Ma, J. and Zhang, X. 2017. Local melatonin application induces cold tolerance in distant organs of Citrullus lanatus L. via long distance transport. Sci. Rep. 7:40858.

Li, S., Xu, Y., Bi, Y., Zhang, B., Shen, S., Jiang, T. and Zheng, X. 2019. Melatonin treatment inhibits gray mold and induces disease resistance in cherry tomato fruit during postharvest. Postharvest Biol. Technol. 157:110962.

Liang, D., Shen, Y., Ni, Z., Wang, Q., Lei, Z., Xu, N., Deng, Q., Lin, L., Wang, J., Lv, X. and Xia, H. 2018. Exogenous melatonin application delays senescence of kiwifruit leaves by regulating the antioxidant capacity and biosynthesis of flavonoids. Front. Plant Sci. 9:426.

Liu, C., Chen, L., Zhao, R., Li, R., Zhang, S., Yu, W., Sheng, J. and Shen, L. 2019. Melatonin induces disease resistance to Botrytis cinerea in tomato fruit by activating jasmonic acid signaling pathway. J. Agric. Food Chem. 67:6116-6124.

Llorens, E., Camañes, G., Lapeña, L. and García-Agustín, P. 2016. Priming by hexanoic acid induce activation of mevalonic and linolenic pathways and promotes the emission of plant volatiles. Front. Plant Sci. 7:495.

Llorens, E., Fernández-Crespo, E., Vicedo, B., Lapeña, L. and García-Agustín, P. 2013. Enhancement of the citrus immune system provides effective resistance against Alternaria brown spot disease. J. Plant Physiol. 170:146-154.

Llorens, E., Vicedo, B., López, M. M., Lapeña, L., Graham, J. H. and García-Agustín, P. 2015. Induced resistance in sweet orange against Xanthomonas citri subsp. citri by hexanoic acid. Crop Prot. 74:77-84.
López-Galiano, M. J., Ruiz-Arroyo, V. M., Fernández-Crespo, E., Rausell, C., Real, M. D., García-Agustín, P., González-Bosch, C. and García-Robles, I. 2017. Oxylipin mediated stress response of a miraculin-like protease inhibitor in hexanoic acid primed eggplant plants infested by Colorado potato beetle. $J$. Plant Physiol. 215:59-64.

Luna, E. 2016. Using green vaccination to brighten the agronomic future. Outlooks Pest Manag. 27:136-140.

Luna, E., Bruce, T. J. A., Roberts, M. R., Flors, V. and Ton, J. 2012. Next-generation systemic acquired resistance. Plant Physiol. 158:844-853.

Martinez-Medina, A., Flors, V., Heil, M., Mauch-Mani, B., Pieterse, C. M. J., Pozo, M. J., Ton, J., van Dam, N. M. and Conrath, U. 2016. Recognizing plant defense priming. Trends Plant Sci. 21:818-822.

Martinez, V., Nieves-Cordones, M., Lopez-Delacalle, M., Rodenas, R., Mestre, T. C., Garcia-Sanchez, F., Rubio, F., Nortes, P. A., Mittler, R. and Rivero, R. M. 2018. Tolerance to stress combination in tomato plants: new insights in the protective role of melatonin. Molecules 23:535.

Mauch-Mani, B., Baccelli, I., Luna, E. and Flors, V. 2017. Defense priming: an adaptive part of induced resistance. Annu. Rev. Plant Biol. 68:485-512.

McElwain, J. C. and Punyasena, S. W. 2007. Mass extinction events and the plant fossil record. Trends Ecol. Evol. 22:548557.

Moustafa-Farag, M., Almoneafy, A., Mahmoud, A., Elkelish, A., Arnao, M. B., Li, L. and Ai, S. 2019. Melatonin and its protective role against biotic stress impacts on plants. Biomolecules 10:54.

Moustafa-Farag, M., Elkelish, A., Dafea, M., Khan, M., Arnao, M. B., Abdelhamid, M. T., El-Ezz, A. A., Almoneafy, A., Mahmoud, A., Awad, M., Li, L., Wang, Y., Hasanuzzaman, M. and Ai, S. 2020. Role of melatonin in plant tolerance to soil stressors: salinity, $\mathrm{pH}$ and heavy metals. Molecules 25:5359.

Niehl, A., Soininen, M., Poranen, M. M. and Heinlein, M. 2018. Synthetic biology approach for plant protection using dsRNA. Plant Biotechnol. J. 16:1679-1687.

Osbourn, A. E. 1996. Preformed antimicrobial compounds and plant defense against fungal attack. Plant Cell 8:1821-1831.

Park, S.-W., Li, W., Viehhauser, A., He, B., Kim, S., Nilsson, A. K., Andersson, M. X., Kittle, J. D., Ambavaram, M. M. R., Luan, S., Esker, A. R., Tholl, D., Cimini, D., Ellerström, M., Coaker, G., Mitchell, T. K., Pereira, A., Dietz, K.-J. and Lawrence, C. B. 2013. Cyclophilin 20-3 relays a 12-oxo-phytodienoic acid signal during stress responsive regulation of cellular redox homeostasis. Proc. Natl. Acad. Sci. U. S. A. 110:9559-9564.

Quintana-Rodriguez, E., Duran-Flores, D., Heil, M. and Camacho-Coronel, X. 2018. Damage-associated molecular patterns (DAMPs) as future plant vaccines that protect crops from pests. Sci. Hortic. 237:207-220.

Ramirez-Prado, J. S., Abulfaraj, A. A., Rayapuram, N., Benhamed, M. and Hirt, H. 2018. Plant immunity: from signaling to epigenetic control of defense. Trends Plant Sci. 23:833-844.

Riemann, M., Dhakarey, R., Hazman, M., Miro, B., Kohli, A. and 
Nick, P. 2015. Exploring jasmonates in the hormonal network of drought and salinity responses. Front. Plant Sci. 6:1077.

Ryan, C. A. and Farmer, E. E. 1991. Oligosaccharide signals in plants: a current assessment. Annu. Rev. Plant Physiol. Plant Mol. Biol. 42:651-674.

Scalschi, L., Vicedo, B., Camañes, G., Fernandez-Crespo, E., Lapeña, L., González-Bosch, C. and García-Agustín, P. 2013. Hexanoic acid is a resistance inducer that protects tomato plants against Pseudomonas syringae by priming the jasmonic acid and salicylic acid pathways. Mol. Plant Pathol. 14:342-355.

Sharif, R., Xie, C., Zhang, H., Arnao, M. B., Ali, M., Ali, Q., Muhammad, I., Shalmani, A., Nawaz, M. A., Chen, P. and Li, Y. 2018. Melatonin and its effects on plant systems. Molecules 23:2352.

Sharma, A. and Zheng, B. 2019. Melatonin mediated regulation of drought stress: physiological and molecular aspects. Plants 8:190.

Shi, H., Chen, K., Wei, Y. and He, C. 2016. Fundamental issues of melatonin-mediated stress signaling in plants. Front. Plant Sci. 7:1124.

Siddiqui, M. H., Alamri, S., Al-Khaishany, M. Y., Khan, M. N., Al-Amri, A., Ali, H. M., Alaraidh, I. A. and Alsahli, A. A. 2019. Exogenous melatonin counteracts $\mathrm{NaCl}$-induced damage by regulating the antioxidant system, proline and carbohydrates metabolism in tomato seedlings. Int. J. Mol. Sci. 20:353.

Simlat, M., Ptak, A., Skrzypek, E., Warchol, M., Morańska, E. and Piórkowska, E. 2018. Melatonin significantly influences seed germination and seedling growth of Stevia rebaudiana Bertoni. PeerJ 6:e5009.

Soukup, M., Martinka, M., Bosnic, D., Caplovicová, M., Elbaum, R. and Lux, A. 2017. Formation of silica aggregates in sorghum root endodermis is predetermined by cell wall architecture and development. Ann. Bot. 120:739-753.

Tan, D.-X., Manchester, L. C., Esteban-Zubero, E., Zhou, Z. and Reiter, R. J. 2015. Melatonin as a potent and inducible endogenous antioxidant: synthesis and metabolism. Molecules 20:18886-188906.

Tan, D.-X., Manchester, L. C., Liu, X., Rosales-Corral, S. A., Acuna-Castroviejo, D. and Reiter, R. J. 2013. Mitochondria and chloroplasts as the original sites of melatonin synthesis: a hypothesis related to melatonin's primary function and evolution in eukaryotes. J. Pineal Res. 54:127-138.

Tan, D.-X. and Reiter, R. J. 2020. An evolutionary view of melatonin synthesis and metabolism related to its biological functions in plants. J. Exp. Bot. 71:4677-4689.

Tauzin, A. S. and Giardina, T. 2014. Sucrose and invertases, a part of the plant defense response to the biotic stresses. Front. Plant Sci. 5:293.

Tepper, C. S. and Anderson, A. J. 1990. Interactions between pectic fragments and extracellular components from the fun- gal pathogen, Colletotrichum lindemuthianum. Physiol. Mol. Plant Pathol. 36:147-158.

Thaler, J. S., Fidantsef, A. L. and Bostock, R. M. 2002. Antagonism between jasmonate- and salicylate-mediated induced plant resistance: effects of concentration and timing of elicitation on defense-related proteins, herbivore, and pathogen performance in tomato. J. Chem. Ecol. 28:1131-1159.

Ton, J. and Mauch-Mani, B. 2004. Beta-amino-butyric acidinduced resistance against necrotrophic pathogens is based on ABA-dependent priming for callose. Plant J. 38:119-130.

Wan, J., Zhang, P., Wang, R., Sun, L., Ju, Q. and Xu, J. 2018. Comparative physiological responses and transcriptome analysis reveal the roles of melatonin and serotonin in regulating growth and metabolism in Arabidopsis. BMC Plant Biol. 18:362.

Wang, N., Wang, L., Zhu, K., Hou, S., Chen, L., Mi, D., Gui, Y., Qi, Y., Jiang, C. and Guo, J.-H. 2019. Plant root exudates are involved in Bacillus cereus AR156 mediated biocontrol against Ralstonia solanacearum. Front. Microbiol. 10:98.

Weeda, S., Zhang, N., Zhao, X., Ndip, G., Guo, Y., Buck, G. A., $\mathrm{Fu}, \mathrm{C}$. and Ren, S. 2014. Arabidopsis transcriptome analysis reveals key roles of melatonin in plant defense systems. PLoS ONE 9:e93462.

Wei, J., Li, D.-X., Zhang, J.-R., Shan, C., Rengel, Z., Song, Z.B. and Chen, Q. 2018. Phytomelatonin receptor PMTR1mediated signaling regulates stomatal closure in Arabidopsis thaliana. J. Pineal Res. 65:e12500.

Wingler, A. 2018. Transitioning to the next phase: the role of sugar signaling throughout the plant life cycle. Plant Physiol. 176:1075-1084.

Yang, X. L., Xu, H., Li, D., Gao, X., Li, T. L. and Wang, R. 2018. Effect of melatonin priming on photosynthetic capacity of tomato leaves under low-temperature stress. Photosynthetica 56:884-892.

Yin, H., Zhao, X. and Du, Y. 2010. Oligochitosan: a plant diseases vaccine-a review. Carbohydr. Polym. 82:1-8.

Zdor, R. E. and Anderson, A. J. 1992. Influence of root colonizing bacteria on the defense responses of bean. Plant Soil 140:99107.

Zhang, N., Zhang, H.-J., Zhao, B., Sun, Q.-Q., Cao, Y.-Y., Li, R., Wu, X.-X., Weeda, S., Li, L., Ren, S., Reiter, R. J. and Guo, Y.-D. 2014. The RNA-seq approach to discriminate gene expression profiles in response to melatonin on cucumber lateral root formation. J. Pineal Res. 56:39-50.

Zhao, D., Yu, Y., Shen, Y., Liu, Q., Zhao, Z., Sharma, R. and Reiter, R. J. 2019. Melatonin synthesis and function: evolutionary history in animals and plants. Front. Endocrinol. 10:249.

Zhao, H., Xu, L., Su, T., Jiang, Y., Hu, L. and Ma, F. 2015. Melatonin regulates carbohydrate metabolism and defenses against Pseudomonas syringae pv. tomato DC3000 infection in Arabidopsis thaliana. J. Pineal Res. 59:109-119. 